\title{
A note on hyperbolic von Mises distributions
}

\author{
JEAN-CLAUDE GRUET \\ Laboratoire de Probabilités et Modèles Aléatoires, Université Pierre et Marie Curie, 4 Place \\ Jussieu, 75252 Paris, France
}

We define hyperbolic von Mises distributions in any integral dimension as exit distribution of hyperbolic Brownian motion $\left(H_{t}^{(\alpha)}, t \geqslant 0\right)$ with drift outside hyperbolic balls centred on the starting point $H_{0}$. Bidimensional unwrapped hyperbolic von Mises distributions are also considered.

Keywords: Brownian motion; hyperbolic; von Mises distribution

\section{Introduction}

Von Mises densities $\operatorname{VM}(k)$ on $[-\pi, \pi]$, given by

$$
\frac{1}{2 \pi I_{0}(k)} \exp (k \cos (\theta))=\frac{1}{2 \pi}+\frac{1}{\pi} \sum_{m=1}^{\infty} \frac{I_{m}(k)}{I_{0}(k)} \cos (m \theta), \quad k \in \mathbb{R}^{+},
$$

were introduced by von Mises (1918) to study the deviations of measured atomic weights from integral values (see Mardia 1972). $I_{m}$ is, of course, the modified Bessel function of the first kind and index $m$. Recall that $\operatorname{VM}(k)$ is the exit law of a complex Brownian motion with drift $\left(Z_{s}+s \mathbf{u}, s \geqslant 0\right)$ starting at 0 outside the circle $x^{2}+y^{2}=1$. Here $k$ denotes the Euclidean norm of the constant vector $\mathbf{u}$. These distributions play a fundamental role in the theory of statistical inference on the circle treated in Mardia (1972) and Watson (1983). Moreover, a maximal entropy characterization in the class of circular distributions on $S^{1}$ was given by Mardia (1972).

Hartman (1976) deduced the infinite divisibility of $\operatorname{VM}(k)$ from general results on linear differential equations and from the existence of a distribution $\mu_{k}$ on $\mathbb{R}^{+}$characterized by its Laplace transform $\lambda \rightarrow I_{\sqrt{2 \lambda}}(k) / I_{0}(k)$. Hence $\operatorname{VM}(k)$ is a mixture of wrapped normal distributions $\mathrm{WN}(\cdot)$ (modulo $2 \pi$ ):

$$
\operatorname{VM}(k)=\int_{0}^{+\infty} \mathrm{WN}(\lambda) \mu_{k}(\mathrm{~d} \lambda)
$$

Kent (1977) gave a different analytical proof of the infinite divisibility, based on the Hartman-Watson mixture property.

These facts were given enlightening probabilistic proofs in Yor (1980) and Pitman and Yor (1981) which we now recall. Let $\left(Z_{t}, 0 \leqslant t \leqslant \tau\right)$ be a planar Brownian bridge of duration $\tau$ starting at a point $z_{0} \neq 0$ and such that $Z_{\tau}=z_{1} \neq 0$. Define its real-valued 
continuous winding process with respect to the origin (not modulo $2 \pi)\left(\Theta_{t}, 0 \leqslant t \leqslant \tau\right)$ such that $\Theta_{0}=0$. Then

$$
\mathbb{E}_{z_{0}}\left[\exp \left(\mathrm{i} u \Theta_{\tau}\right) / Z_{\tau}=z_{1}\right]=\frac{I_{|u|}(r)}{I_{0}(r)}, \quad r=\frac{\left|z_{0} z_{1}\right|}{\tau} .
$$

As usual, $\mathbb{E}_{z_{0}}$ denotes the expectation under the law of the process started at $z_{0}$. Hence, the skew product decomposition of planar Brownian motion enables us to rewrite (1.1) with the help of the bracket $\langle\Theta\rangle_{t}=\int_{0}^{t} \mathrm{~d} s /\left|Z_{s}\right|^{2}$. Thus if $\left(\rho_{t}, 0 \leqslant t \leqslant \tau\right)$ is a Bessel bridge with index 0 such that $\rho_{0}=a>0$ and $\rho_{\tau}=b>0$, the Laplace transform of the probability density of $\int_{0}^{\tau} \rho_{s}^{-2} \mathrm{~d} s$ is precisely $I_{\sqrt{2 \lambda}}(r) / I_{0}(r)$, where $r=a b / \tau$.

Note that the right-hand side of (1.1) is roughly the quotient of densities of Bessel processes with indices $|u|$ and 0 at time $r$. Indeed, the probability density function (pdf) of a $v$ Bessel process $\operatorname{Bes}(v)$ with respect to the Lebesgue measure is

$$
p_{t}(x, y)=\frac{y}{t}\left(\frac{y}{x}\right)^{v} \exp \left(\frac{-\left(x^{2}+y^{2}\right)}{2 t}\right) I_{v}\left(\frac{x y}{t}\right),
$$

provided that $x$ and $t$ are positive. When $d=2 v+2$ is an integer, Bessel processes are simply the Euclidean norm of $d$-dimensional Brownian motions. Yor (1980) explained by means of Girsanov's theorem why the pdf of a $\operatorname{Bes}(v)$ process at time $t$ is linked with the conditional characteristic function in (1.1). We show in Gruet (1997) that more generally the conditional characteristic function of a winding in a two-point homogeneous symmetric space of dimension 2 is proportional to the quotient of the pdf corresponding to the associated Bessel processes with indices $|u|$ and 0 .

A more pleasant representation of $I_{|u|}(r) / I_{0}(r)$ which does not use a Brownian bridge is given in Pitman and Yor (1981). Consider $\left(\rho_{s}, s \geqslant 0\right)$ a Bessel process of index 0 with drift $\delta$, namely the modulus of $Z_{t}+\delta t$ where $\left(Z_{t}\right)$ is a complex Brownian motion starting at 0 . Denote its law by $\mathbb{P}^{\delta}$, where $\delta$ is positive. Then if $T_{r}$ is the first hitting time of $r$, the time inversion $Z_{t} \rightarrow t Z_{1 / t}$ yields

$$
\mathbb{E}_{0}^{\delta}\left(\exp \left(-\lambda \int_{T_{r}}^{+\infty} \frac{\mathrm{d} s}{\rho_{s}^{2}}\right)\right)=\frac{I_{\sqrt{2 \lambda}}(\delta r)}{I_{0}(\delta r)} .
$$

In this paper we consider some hyperbolic analogues of these results. Our aim is to discuss whether the fine properties of Euclidean Brownian motion - the scaling property, stability by time inversion, etc. - are essential for solving these problems. Therefore, we consider the simplest rotationally symmetric non-Euclidean Riemannian space, that is to say, the real hyperbolic space with constant curvature -1 . Some structural insight should be gained by the isolation of geometric properties from analytical considerations on secondorder differential operators.

An essentially different hyperbolic analogue of the Euclidean von Mises law was defined by Barndorff-Nielsen (1978): he obtained a law on the entire hyperbolic $d$-dimensional space, absolutely continuous with respect to the volume element. The analogy with the Euclidean case raised statistical problems discussed by Jensen (1981) and Casalis et al. 
(1993). We believe that our hyperbolic von Mises laws could play some role in the study of directional data on hyperbolic spaces. This geometry is relevant in a lot of physical questions: for instance, radio waveguides in Gertsenshtein and Vasil'ev (1959) and diffusions in random media in Comtet and Monthus (1996). Note that hyperbolic Brownian motion with vertical drift is used in the latter paper. Hyperbolic heat kernels with uniform magnetic field are discussed by Ikeda and Matsumoto (1999).

This paper is organized as follows. In Section 2, we treat the simpler two-dimensional case. In Section 3, we briefly consider the multidimensional case $d=2 v+2$ which makes sense even for positive real-valued $\nu$.

\section{The two-dimensional case}

\subsection{Some background on hyperbolic planar geometry}

We will use the Poincaré half-space model: $\mathbb{U}=\mathbb{R} \times \mathbb{R}^{+}$is equipped with its hyperbolic metric

$$
\mathrm{d} s^{2}=\frac{\mathrm{d} x^{2}+\mathrm{d} y^{2}}{y^{2}}
$$

and its Laplace-Beltrami operator $\Delta_{\mathbb{H} \text {. The hyperbolic Brownian motion infinitesimal }}$ generator is

$$
\mathscr{G}^{0}=\frac{1}{2} \Delta_{\mathbb{\Downarrow}}=\frac{1}{2} y^{2}\left(\frac{\partial^{2}}{\partial x^{2}}+\frac{\partial^{2}}{\partial y^{2}}\right) .
$$

More precisely, if $\mathbf{B}=\left(B_{t}^{(1)}, B_{t}^{(2)}\right)$ denotes a two-dimensional Euclidean Brownian motion, then the unique (strong) solution of the system

$$
\begin{aligned}
& X_{t}=x+\int_{0}^{t} Y_{s} \mathrm{~d} B_{s}^{(1)} \\
& Y_{t}=y+\int_{0}^{t} Y_{s} \mathrm{~d} B_{s}^{(2)} .
\end{aligned}
$$

is referred to as B-hyperbolic Brownian motion $\left(H_{t}=\left(X_{t}, Y_{t}\right), t \geqslant 0\right)$ starting at $p=(x, y) \in \mathbb{H}$.

Next, we define the generator $\mathscr{G}^{(\alpha)}$ of hyperbolic Brownian motion with drift $\alpha$ along the $y$-axis by adding $\alpha y \partial / \partial y$ to $\mathscr{G}^{0}$. Hence the second component becomes

$$
Y_{t}^{(\alpha)}=y \exp \left(B_{t}^{(2)}-\frac{t}{2}+\alpha t\right),
$$

and there exists a linear Brownian motion $\left(\gamma_{t}, t \geqslant 0\right)$ independent of $B^{(2)}$ such that

$$
X_{t}^{(\alpha)}=x \gamma\left(A_{t}^{(v)}\right)
$$


where $A_{t}^{(v)}$ denotes the exponential functional $\int_{0}^{t} \exp \left(2\left(B_{s}^{(2)}+v s\right)\right) \mathrm{d} s$ with $v=\alpha-\frac{1}{2}$.

\subsection{Hyperbolic Brownian motion with drift}

We first explain why hyperbolic Brownian motion with drift is the hyperbolic analogue of the well-known Euclidean object.

A hyperbolic translation $T$ should be an isometry without finite fixed points and such that at least one geodesic is globally invariant. If the geodesic is supported by the $y$-axis, obviously $T(z)=q^{2} z$ with $q^{2} \neq 0, q^{2} \neq 1$. We will say that the translation is vertical. Observe that the second component of hyperbolic Brownian motion with $\operatorname{drift}\left(H_{t}^{(\alpha)}, t \geqslant 0\right)$ defined above has the same law as $\left(\mathrm{e}^{\alpha t} Y_{t}, t \geqslant 0\right)$.

More generally, a hyperbolic translation is a linear map $z \rightarrow(a z+b) /(c z+d)$ associated with a matrix conjugate to

$$
\left(\begin{array}{cc}
q & 0 \\
0 & 1 / q
\end{array}\right)
$$

with $q \neq 0$ and $q \neq 1$. These elements are called hyperbolic in the literature (see Terras 1985). From our point of view, parabolic isometries (i.e. conjugate to $z \rightarrow z+c$ for some real c) are not the natural hyperbolic translations.

Secondly, if $\mathbb{P}^{(\alpha)}$ denotes the law of $\left(H_{t}^{(\alpha)}, t \geqslant 0\right)$ on the canonical space $\mathscr{C}\left(\mathbb{R}^{+}, \mathbb{H}\right)$ endowed with its natural filtration $\left(\mathscr{F}_{t}\right)_{t \geqslant 0}$, we readily obtain, as a consequence of Girsanov's theorem,

$$
\frac{\mathrm{dP}^{(\alpha)}}{\mathrm{d} \mathbb{P}^{(0)}}=D_{t} \quad \text { on } \mathscr{F}_{t}
$$

for the positive martingale $D_{t}=\left(Y_{t} / y\right)^{\alpha} \exp (-\alpha(\alpha-1) t / 2)=\exp \left(\alpha B_{t}^{(2)}-\left(\alpha^{2} / 2\right) t\right)$. Indeed, $\left.h_{\alpha}(t, x, y)=y^{\alpha} \exp (-\alpha(\alpha-1) t / 2)\right)$ solves the equation $\partial h_{\alpha} / \partial t+\mathscr{G}^{0} h_{\alpha}=0$. In real analysis such a function is called parabolic, whereas in probability theory Yor (1992), in a Euclidean setting, called it a space-time $\mathscr{G}^{0}$ harmonic function. For the rest of this section we write $\lambda=\frac{1}{2} \alpha(\alpha-1)$.

We recall next the construction of the hyperbolic polar coordinates $(r, u) \in \mathbb{R}^{+} \times \mathbb{R} / 2 \pi \mathbb{Z}$ with centre $i$ :

$$
x=\frac{\sinh (r) \sin (u)}{\cosh (r)+\cos (u) \sinh (r)}, \quad y=\frac{1}{\cosh (r)+\cos (u) \sinh (r)} .
$$

Note that we use $u$ in the same way as Rogers and Williams (1987, p. 214) instead of the classical $2 u$ in Helgason (1984) or Terras (1985). Hence we can define a polar decomposition $\left(\left(R_{t}, U_{t}\right), t \geqslant 0\right)$ for every continuous nice $(\mathbb{H}-\{i\})$-valued process.

We will need some basic facts on Legendre functions of the first kind, $P_{-\alpha}^{(m)}$. For any nonnegative integer $m$ and any $\alpha<1$, we have, from formula 3.7(14) in Bateman et al. (1953),

$$
P_{-\alpha}^{(m)}(\cosh (r))=\frac{\Gamma(m+1-\alpha)}{\pi \Gamma(1-\alpha)} \int_{0}^{\pi}(\cosh (r)+\sinh (r) \cos (\theta))^{-\alpha} \cos (m \theta) \mathrm{d} \theta .
$$


If $\alpha>0$, we use Bateman et al. (1953, 3.7 (13)): for every non-negative integer $m$,

$$
P_{-\alpha}^{(-m)}(\cosh (r))=\frac{\Gamma(\alpha)}{\pi \Gamma(m+\alpha)} \int_{0}^{\pi}(\cosh (r)+\sinh (r) \cos (\theta))^{-\alpha} \cos (m \theta) \mathrm{d} \theta .
$$

Definition. Let $T_{r}$ be the first hitting time of the hyperbolic circle of radius $r$, centred at $i$. The probability distribution of $U\left(T_{r}\right)$ under the law $\mathbb{P}_{i}^{(\alpha)}$ of the process $\left(H_{t}^{(\alpha)}, t \geqslant 0\right)$, starting at $i$, is called the two-dimensional hyperbolic von Mises distribution $\Lambda^{\alpha}(r, \mathrm{~d} u)$ with parameter $\alpha$.

Proposition 1. The following explicit formula holds for any $\alpha$ :

$$
\Lambda^{\alpha}(r, \mathrm{~d} u)=\frac{1}{2 \pi P_{-\alpha}^{(0)}(\cosh (r))}(\cosh (r)+\sinh (r) \cos (u))^{-\alpha_{\llbracket}[-\pi, \pi]}[u) \mathrm{d} u,
$$

where $P_{-\alpha}^{(0)}$ denotes the Legendre function of index 0 .

Corollary 2. (A Schlömilch-like formula). If $\alpha<1$,

$$
\Lambda^{\alpha}(r, \mathrm{~d} u)=\frac{\mathrm{d} u}{2 \pi}+\frac{1}{\pi P_{-\alpha}^{(0)}(\cosh (r))}\left(\sum_{m \geqslant 1} \frac{\Gamma(1-\alpha)}{\Gamma(m+1-\alpha)} P_{-\alpha}^{(m)}(\cosh (r)) \cos (m u)\right) \mathrm{d} u
$$

If $\alpha>0$,

$$
\Lambda^{\alpha}(r, \mathrm{~d} u)=\frac{\mathrm{d} u}{2 \pi}+\frac{1}{\pi P_{-\alpha}^{(0)}(\cosh (r))}\left(\sum_{m \geqslant 1} \frac{\Gamma(m+\alpha)}{\Gamma(\alpha)} P_{-\alpha}^{(-m)}(\cosh (r)) \cos (m u)\right) \mathrm{d} u .
$$

Proof. By the absolute continuity result (2.5) and (2.6) we obtain

$$
\Lambda^{\alpha}(r, \mathrm{~d} u)=\mathbb{E}_{i}\left(\exp \left(-\lambda T_{r}\right)\right) \frac{1}{2 \pi}(\cosh (r)+\sinh (r) \cos (u))^{-\alpha} \mathrm{d} u .
$$

Obviously under the law $\mathbb{P}_{i}^{(0)} U\left(T_{r}\right)$ is uniformly distributed on $[-\pi, \pi]$. If $\left(R_{t}\right)$ denotes the radial part of the ordinary hyperbolic Brownian motion, we deduce from the integral representation (2.7) that $\left(P_{\alpha}^{(0)}\left(\cosh \left(R_{t \wedge T_{r}}\right)\right) \exp \left(-\lambda\left(t \wedge T_{r}\right)\right), t \geqslant 0\right)$ is a bounded martingale. Hence $\mathbb{E}_{i}\left(\exp \left(-\lambda T_{r}\right)\right)=1 / P_{-\alpha}^{(0)}(\cosh (r))$.

Consider for $\alpha>1$ the increasing solution of $\mathscr{G} \varphi=\lambda \varphi=(\alpha(\alpha-1) / 2) \varphi$,

$$
r \rightarrow P_{-\alpha}^{(0)}(\cosh (r))=\int_{S_{r}} y^{\alpha} \mathrm{d} \sigma(z),
$$

where $S_{r}$ is the hyperbolic circle of radius $r$ centred at $i$ and $\mathrm{d} \sigma$ the hyperbolic normalized surface measure. Indeed, $(x, y) \rightarrow y^{\alpha}$ is hyperbolically subharmonic. Hence, we will also call this function $\phi_{\lambda}^{\uparrow}(r)$ when we want to stress the monotonicity property. The use of down and up arrows is taken from Pitman and Yor's (1981) study of conditioned diffusions. 


\section{Remarks.}

(i) Note that Terras (1985, Exercise 9, p. 141) erroneously refers to Lebedev (1972) who does not consider (associated) Legendre functions $P_{\alpha}^{(m)}$ with non-integer indices $m$. In fact, the well-known formula (2.7) when $m$ is integer was generalized by Erdélyi (1941) to real numbers. But this generalized formula takes a different form. Beware of the fact that $P_{A}^{-(v)}$ and $P_{A}^{(v)}$ are proportional if and only if $v$ is an integer (Bateman et al. 1953, 3.3.1 (6)).

(ii) J. Kent remarked in the discussion following Mardia (1975) that the von Mises law can be considered as the invariant probability of what he called an Ornstein-Uhlenbeck process on the circle. Such a Markov process may be defined via its infinitesimal generator

$$
\frac{1}{2} \frac{\partial^{2}}{\partial \theta^{2}}-k \sin (\theta) \frac{\partial}{\partial \theta}
$$

We could define for the same purpose hyperbolic Ornstein-Ulhenbeck processes on the circle by their generators

$$
\frac{1}{2}\left(\frac{\partial^{2}}{\partial \theta^{2}}+\frac{\alpha \sinh (r) \sin (\theta)}{\cosh (r)+\sinh (r) \cos (\theta)} \frac{\partial}{\partial \theta}\right) .
$$

(iii) $\left(H_{t}^{(1)}, t \geqslant 0\right)$ is the process $\left(H_{t}, t \geqslant 0\right)$ conditioned to hit the boundary $\partial \mathbb{H}$ at $\infty$ in Doob's sense.

(iv) Euclidean $\operatorname{VM}(-k)$ is the image of $\operatorname{VM}(k)$ by the half-turn $z \mapsto-z$. We see from Proposition 1 that, unfortunately, the image of $\Lambda^{\alpha}(r, \mathrm{~d} u)$ by the hyperbolic half-turn $z \mapsto I(z)=-1 / z$ (or $(r, u) \mapsto(r, u+\pi)$ in geodesic polar coordinates) is not a hyperbolic von Mises distribution. In fact, the $\left(I\left(H_{t}^{(0)}\right), t \geqslant 0\right)$ law is absolutely continuous with respect to the $\left(H_{t}, t \geqslant 0\right)$ law. Expression (2.5) still holds if the function $(x, y) \mapsto y$ is replaced by the harmonic function $(x, y) \mapsto h_{1}(t, x, y)=y /\left(x^{2}+y^{2}\right)$.

By a standard martingale argument we readily obtain the following:

Corollary 3. Let $Q$ be the Legendre function of the second kind and $A(\lambda)=\frac{1}{2}(1-\sqrt{1+8 \lambda})$. Write $\phi_{\lambda}^{\uparrow}(r)=P_{-A(\lambda)}^{(0)}(r)$ and $\phi_{\lambda}^{\downarrow}(r)=Q_{-A(\lambda)}^{(0)}(r)$ for the fundamental solutions of $\mathscr{G} \varphi=\lambda \varphi$. Then, for every non-negative $\mu$, if $0<a<r$,

$$
\mathbb{E}_{a}^{(a)}\left(\exp \left(-\mu T_{r}\right)\right)=\frac{\phi_{\lambda}^{\uparrow}(r)}{\phi_{\lambda}^{\uparrow}(a)} \frac{\phi_{\lambda+\mu}^{\uparrow}(a)}{\phi_{\lambda+\mu}^{\uparrow}(r)},
$$

whereas if $a \geqslant r$,

$$
\mathbb{E}_{a}^{(\alpha)}\left(\exp \left(-\mu T_{r}\right)\right)=\frac{\phi_{\lambda}^{\uparrow}(r)}{\phi_{\lambda}^{\uparrow}(a)} \frac{\phi_{\lambda+\mu}^{\downarrow}(a)}{\phi_{\lambda+\mu}^{\downarrow}(r)},
$$

where, as usual, $\mathbb{E}_{a}^{\alpha}$ denotes the expectation under the law of a hyperbolic Bessel process with positive drift $\alpha$ starting at $a$, and $\lambda=\frac{1}{2} \alpha(\alpha-1)$. 
Proof. We use Proposition 3.1 in Pitman and Yor (1981): we have on the trace of $\mathscr{F}_{T_{r}}$ by the event $\left\{T_{r}<\infty\right\}$,

$$
\frac{\mathrm{d} \mathbb{P}_{a}^{(\alpha)}}{\mathrm{dP}_{a}^{(0)}}=\exp \left(-\lambda T_{r}\right) \frac{\phi_{\lambda}^{\uparrow}\left(X_{T_{r}}\right)}{\phi_{\lambda}^{\uparrow}(a)}
$$

Hence

$$
\mathbb{E}_{a}^{(\alpha)}\left(\exp \left(-\mu T_{r}\right)\right)=\mathbb{E}_{a}\left(\exp \left(-(\lambda+\mu) T_{r}\right)\right) \frac{\phi_{\lambda}^{\uparrow}(r)}{\phi_{\lambda}^{\uparrow}(a)} .
$$

We conclude with the help of the appropriate stopped martingale.

An easy consequence of the absolute continuity (2.5) is the hyperbolic translation of Wendel's (1980) independence result:

Theorem 4. Denote by $T_{r}$ the first hitting time of the hyperbolic circle of radius $r$ centred at the starting point $H_{0}^{(\alpha)}$. Then, for every $\alpha, T_{r}$ and $H^{(\alpha)}\left(T_{r}\right)$ are independent. Moreover, the radial part $\left(R_{t}, t \leqslant T_{r}\right)$ and the exit point are independent.

Proof. Remark that the density $D\left(T_{r}\right)$ is the product of a $\sigma\left(R_{s}^{(\alpha)}, s \leqslant T_{r}\right)$ measurable random variable by a $\sigma\left(H^{(\alpha)}\left(T_{r}\right)\right)$ one and then apply Lemma 5.1 in Pitman and Yor (1981).

A remark about Barndorff-Nielsen's hyperboloid law. Consider the well-known isometry

$$
\left(X_{1}, X_{2}, X_{3}\right) \rightarrow \frac{\left(X_{2}, X_{3}\right)}{X_{1}}
$$

between the upper half-hyperboloid $-X_{1}^{2}+X_{2}^{2}+X_{3}^{2}=1$ and the unit disc. If we translate Barndorff-Nielsen's definition from the hyperboloid to Poincare half-plane setting, we obtain the radial density $\exp (\kappa) \kappa \exp (-\kappa \cosh (r))$ with respect to the hyperbolic volume element $(1 / 2 \pi) \sinh (r) \mathrm{d} r \mathrm{~d} u$ on $\mathbb{H}$. Here $\kappa$ is a positive parameter. Hence the hyperboloid law has more to do with the three-dimensional von Mises density of Fisher $(1953)$ on $[0, \pi]$, $(k / 2 \sinh (k)) \exp (k \cos (\varphi)) \sin (\varphi)$.

\subsection{Hyperbolic Bessel processes with drift}

Denote by $\left(R_{t}\right)$ the radial part of $\left(H_{t}\right)$ : this is a time-homogeneous diffusion process called a hyperbolic Bessel process of index $v=0$. The corresponding infinitesimal generator is half the radial part of the hyperbolic Laplacian:

$$
\frac{1}{2}\left(\frac{\partial^{2}}{\partial r^{2}}+\operatorname{coth}(r) \frac{\partial}{\partial r}\right)
$$

(for a similar result, see Rogers and Williams 1987). Its transition density with respect to the Lebesgue measure is written in Gruet (1997): 


$$
q_{2}(t, x, y)=\sinh (y) \int_{0}^{+\infty} \exp \left(-\left(\frac{1}{4}+p^{2}\right) \frac{t}{2}\right) P_{-\frac{1}{2}+i p}^{(0)}(\cosh (x)) P_{-\frac{1}{2}+i p}^{(0)}(\cosh (y)) p \tanh (\pi p) \mathrm{d} p
$$

When $x=0$, the following well-known formula is obtained:

$$
q_{2}(t, 0, y)=\sinh (y) \pi^{-1 / 2} t^{-3 / 2} \exp \left(-\frac{t}{8}\right) \int_{y}^{+\infty} \frac{u \exp \left(-u^{2} / 2 t\right)}{\sqrt{\cosh (u)-\cosh (y)}} \mathrm{d} u .
$$

Although several integral representations are listed in Gruet $(1996 ; 1997)$, these densities cannot be much simplified.

The results of Pitman and Rogers (1981) on Markov functions imply the following:

Theorem 5. Let $H^{(\alpha)}$ be a hyperbolic Brownian motion with a vertical drift of magnitude $\alpha$, starting at the origin $i$. Then the radial part $R$ is a time-homogeneous diffusion process on $[0, \infty)$ with transition density $\exp \left(-\frac{1}{2} \alpha(\alpha-1) t\right) q_{2}(t, x, y) h_{2}(\alpha, y) / h_{2}(\alpha, x)$.

\subsection{Unwrapped hyperbolic von Mises distribution}

Let $f(x)$ be a candidate probability density on $\mathbb{R}$ for an unwrapped hyperbolic von Mises density with parameters $\alpha$ and $r>0$, namely

$$
\sum_{k} f(x+2 k \pi)=\Lambda^{\alpha}(r, x)
$$

this yields, by the Poisson summation formula,

$$
\hat{f}(m)=\int_{0}^{2 \pi} \cos (m u) \Lambda^{\alpha}(r, \mathrm{~d} u)=\pi a_{m}
$$

where $a_{m}$ is the Fourier cosine coefficient $(m \neq 0)$. If $\alpha>0$, we obtain

$$
\pi a_{m}=\frac{P_{-\alpha}^{(-m)}(\cosh (r))}{P_{-\alpha}^{(0)}(\cosh (r))} \frac{\Gamma(m+\alpha)}{\Gamma(\alpha)} .
$$

Thus a candidate would be the even function $\phi_{\alpha}$ such that, for every positive $\lambda$,

$$
\phi_{\alpha}(\lambda)=\frac{\Gamma(\lambda+\alpha)}{\Gamma(\alpha)} \frac{P_{-\alpha}^{(-\lambda)}(\cosh (r))}{P_{-\alpha}^{(0)}(\cosh (r))} .
$$

The hyperbolic analogue of the series which defines the $\operatorname{VM}(k)$ density is

$$
\frac{1}{2 \pi}+\frac{1}{\pi} \sum_{m=1}^{\infty} \frac{\Gamma(m+\alpha)}{\Gamma(\alpha)} \frac{P_{-\alpha}^{(-m)}(\cosh (r))}{P_{-\alpha}^{(0)}(\cosh (r))} \cos (m \theta) .
$$

Theorem 6. If $\alpha>0$, the function

$$
\lambda \rightarrow \frac{\Gamma(\sqrt{2 \lambda}+\alpha)}{\Gamma(\alpha)} \frac{P_{-\alpha}^{(-\sqrt{2 \lambda})}(\cosh (r))}{P_{-\alpha}^{(0)}(\cosh (r))},
$$


defined on $\mathbb{R}^{+}$, is the Laplace transform of a probability $\eta_{\alpha, r}$ on $\mathbb{R}^{+}$. Hence, every unwrapped hyperbolic von Mises law with positive parameter $\alpha$ is a mixture of Gaussian laws.

Remark. When the curvature $-c$ of the hyperbolic space increases to zero, this hyperbolic unwrapped von Mises distribution becomes the usual unwrapped von Mises distribution. The metric becomes

$$
\left(\begin{array}{cc}
1 & 0 \\
0 & A^{2}(r)
\end{array}\right)
$$

with $A(r)=(1 / c) \sinh (c r)$ and $r$ is multiplied by $c$.

The following identity deduced from Bateman et al. (1953, 3.7 (13)) yields

$$
\begin{gathered}
P_{-\alpha}^{(0)}(\cosh (r)) \phi_{\alpha}\left(\frac{\lambda^{2}}{2}\right)=\frac{1}{\pi} \int_{0}^{\pi}\left(\frac{1}{\cosh (r)-\sinh (r) \cos (u)}\right)^{\alpha} \cos (\lambda u) \mathrm{d} u \\
-\frac{\sin (\lambda \pi)}{\pi} \int_{0}^{+\infty}\left(\frac{1}{\cosh (r)+\sinh (r) \cosh (u)}\right)^{\alpha} \exp (-\lambda u) \mathrm{d} u
\end{gathered}
$$

if $\alpha>0$ and $\lambda \geqslant 0$. This is the hyperbolic counterpart of the identity

$$
I_{\lambda}(r)=\frac{1}{\pi} \int_{0}^{\pi} \mathrm{e}^{r \cos (\theta)} \cos (\lambda \theta) \mathrm{d} \theta-\frac{\sin (\lambda \pi)}{\pi} \int_{0}^{+\infty} \mathrm{e}^{-r \cosh (t)-\lambda t} \mathrm{~d} t, \quad \text { for } \lambda \geqslant 0 .
$$

Hence, if $r$ is replaced by $c r$ with $c$ going to zero, we check that $P_{-\alpha / c}^{(0)}(\cosh (c r)) \phi_{\alpha / c}\left(\lambda^{2} / 2\right)$ goes to $I_{\lambda}(\alpha r)$. Hence $\phi_{\alpha / c}\left(\lambda^{2} / 2\right)$ goes to $I_{\lambda}(\alpha r) / I_{0}(\alpha r)$.

Proof. Although this result is sketched in Hartman (1976, p. 272), we give some details.

We apply Theorem 1.2 of Hartman (1976). We choose $\sigma=+\infty$ and the principal solution at 1 , defined on $T=] 1,+\infty]$,

$$
\xi(x, \mu)=P_{-\alpha}^{(-\sqrt{2 \mu})}(x) \Gamma(\alpha+\sqrt{2 \mu})
$$

of the Legendre equation

$$
\left(1-x^{2}\right) w^{\prime \prime}(x)-2 x w^{\prime}(x)+\left((\alpha-1) \alpha-\frac{2 \mu}{1-x^{2}}\right) u(x)=0 .
$$

Then Hartman's hypothesis $(A .3)_{\sigma}$,

$$
\text { for every } \mu, v \geqslant 0, \lim _{x \rightarrow+\infty} \frac{\xi(x, \mu)}{\xi(x, v)}=1,
$$

is satisfied and $q(x, \mu)=2 \mu\left(1-x^{2}\right)^{-2}+\alpha(\alpha-1)\left(x^{2}-1\right)^{-1}$ has a positive partial derivative in $\mu$.

Corollary 7. If $\alpha>0$, the unwrapped hyperbolic von Mises law and wrapped hyperbolic von Mises law are infinitely divisible. 
Proof. The idea is also borrowed from Hartman. His Theorem 1.1 implies, for any $0<t<\tau$, the existence of a measure $W(\mathrm{~d} r, t, \tau)$ on $\mathbb{R}^{+}$such that, for every $\lambda \geqslant 0$,

$$
\frac{\xi(\tau, \lambda) \xi(t, 0)}{\xi(\tau, 0) \xi(t, \lambda)}=\int_{0}^{+\infty} \exp (-\lambda r) W(\mathrm{~d} r, t, \tau)
$$

Obviously, if $\left(\tau_{k}\right)$ is an increasing sequence,

$$
W\left(\cdot, \tau_{1}, \tau_{n}\right)=W\left(\cdot, \tau_{1}, \tau_{2}\right) * \ldots * W\left(\cdot, \tau_{n-1}, \tau_{n}\right)
$$

and $W(r, t, \tau) \rightarrow \delta_{0}(r)$ as $t, \tau \rightarrow 0$. Then $W(\cdot, t, \tau)$ is infinitely divisible. But from hypothesis $(A .3)_{\sigma}, W(\cdot, t, \tau)$ narrowly converges to $\eta_{\alpha, r}$ when $\tau$ increases to infinity.

\section{Remarks.}

(i) We are unable to find a probabilistic proof of these results because we do not know a family of diffusion processes with a kernel proportional to $P_{\alpha}^{(-v)}(\cosh (r))$ such as the Bessel processes in the Euclidean case. For instance, hyperbolic Bessel processes, associated with

$$
\frac{1}{2}\left(\frac{\partial^{2}}{\partial r^{2}}+(2 v+1) \operatorname{coth}(r) \frac{\partial}{\partial r}\right)
$$

have complicated pdf; for the whole story, we refer to our previous work on hyperbolic windings (Gruet, 1997). Contrary to the Euclidean situation, hyperbolic von Mises distributions do not have obvious connections with hyperbolic Bessel processes of index different from 0 .

(ii) Moreover, Watanabe (1975) showed that if a diffusion process on the half-line enjoys the time-inversion property, then this is a homomorphism of a Euclidean Bessel process with drift. Of course, hyperbolic Bessel processes do not share this property.

We compute now the density of $\eta_{\alpha, r}$ which looks very similar to the ordinary HartmanWatson one. Note that, due to the lack of scaling, two parameters $r$ and $\alpha$ are required.

Theorem 8. The hyperbolic Hartman-Watson law has a density on $\mathbb{R}^{+}$,

$$
\eta_{\alpha, r}(u)=\frac{1}{\pi^{3 / 2}(2 u)^{1 / 2}} \exp \left(\frac{\pi^{2}}{2 u}\right) \frac{\sinh (r)}{P_{-\alpha}^{(0)}(\cosh (r))} \Psi_{\alpha, r}(u),
$$

with

$$
\Psi_{\alpha, r}(u)=\int_{0}^{+\infty} \exp \left(\frac{-x^{2}}{2 u}\right) \frac{\alpha \sinh (x)}{(\cosh (r)+\sinh (r) \cosh (x))^{\alpha+1}} \sin \left(\frac{\pi x}{u}\right) \mathrm{d} x .
$$

Proof. We mimic Yor's (1980) proof for the Euclidean analogue (Theorem 5.4(ii)). Hence we will skip some details, the use of Fubini's theorem for instance. We will consider the same paths $C_{u}$ in the complex plane supported by the boundary of an infinite rectangle with vertices $-\mathrm{i} \pi+\infty,-\mathrm{i} \pi+u,+\mathrm{i} \pi+u$ and $\mathrm{i} \pi+\infty$ (see Yor's Fig. 1). From (2.8), we have 


$$
P_{-\alpha}^{(0)}(\cosh (r)) \phi_{\alpha}(\lambda)=\frac{1}{2 \mathrm{i} \pi} \int_{C_{0}}\left(\frac{1}{\cosh (r)-\sinh (r) \cosh (\omega)}\right)^{\alpha} \exp (-\sqrt{2 \lambda} \omega) \mathrm{d} \omega .
$$

With the help of the well-known identity,

$$
\exp (-\sqrt{2 \lambda} x)=\int_{0}^{+\infty} \exp (-\lambda u) \frac{1}{\left(2 \pi u^{3}\right)^{1 / 2}} x \exp \left(-x^{2} / 2 u\right) \mathrm{d} u,
$$

we obtain that $\eta_{\alpha, r}$ has the density $\left(P_{-\alpha}^{(0)}(\cosh (r))\right)^{-1}\left(2 \pi u^{3}\right)^{-1 / 2} H(u)$, where

$$
H(u) \stackrel{(\text { def })}{=} \frac{1}{2 \mathrm{i} \pi} \int_{C_{0}}(\cosh (r)-\sinh (r) \cosh (\omega))^{-\alpha} \omega \exp \left(-\omega^{2} / 2 u\right) \mathrm{d} \omega .
$$

Now $2 \pi H(u)$ may be written in the form

$$
\int_{0}^{+\infty}\left[(-2 x \sin (\pi x / u)+2 \pi \cos (\pi x / u)] \exp \left(\left(\pi^{2}-x^{2}\right) / 2 u\right)(\cosh (r)+\sinh (r) \cosh (x))^{-\alpha} \mathrm{d} x,\right.
$$

so that

$$
H(u)=\frac{1}{\pi} \exp \left(\pi^{2} / 2 u\right) \int_{0}^{+\infty} \exp \left(-x^{2} / 2 u\right) \frac{\alpha u \sinh (r) \sinh (x)}{(\cosh (r)+\sinh (r) \cosh (x))^{\alpha+1}} \sin (\pi x / u) \mathrm{d} x
$$

follows from integration by parts.

We are now in a position to obtain an interesting consequence, namely to find a second hyperbolic analogue of Hartman-Watson law:

Corollary 9. If $\alpha$ is big enough, then $\lambda \rightarrow P_{-\alpha}^{(-\lambda)}(\cosh (r)) / P_{-\alpha}^{(0)}(\cosh (r))$ is the Laplace transform of a probability density on $\mathbb{R}^{+}$taken at $\lambda^{2} / 2$.

Proof. In fact, we show that $\lambda \rightarrow \Gamma(\alpha) / \Gamma(\alpha+\sqrt{2 \lambda})$ is a Laplace transform, provided $S \stackrel{\text { (def) }}{=} \gamma-\alpha \sum_{k=1}^{+\infty} 1 / k(k+\alpha)+1 / \alpha$ is negative.

Note that $\lim _{\alpha \rightarrow \infty} \alpha \sum_{k=1}^{+\infty} 1 / k(k+\alpha)=\infty$. On the other hand, when $\alpha$ is too small we see by an obvious monotonicity inspection that $\Gamma(\alpha) / \Gamma(\alpha+\sqrt{2 \lambda})$ is definitely not a decreasing function on the positive half-line.

We use the following facts collected in Yor (1993, Proposition 5):

(i) The Weierstrass product formula: if $\gamma$ denotes the Euler constant,

$$
\frac{\exp (-\gamma z)}{\Gamma(z)}=z \prod_{k=1}^{\infty}\left(1+\frac{z}{k}\right) \exp \left(-\frac{z}{k}\right) \text {. }
$$

(ii) The Laplace transform of the probability density $(1 / \sqrt{2 \pi}) x^{-5 / 2} \exp (-1 / 2 x) \rrbracket_{\mathbb{R}^{+}}(x) \mathrm{d} x$ is

$$
\lambda \rightarrow h(\lambda) \stackrel{(\text { def })}{=}(1+\sqrt{2 \lambda}) \exp (-\sqrt{2 \lambda}) .
$$


(iii) For every positive $C$, there exists a stable positive random variable $T_{C}$ of index $\frac{1}{2}$ such that $\exp (-C|\lambda|)=\mathbb{E}\left(\exp \left(-\lambda^{2} / 2 T_{C}\right)\right)$.

Observe now that $\Gamma(\alpha) / \Gamma(\alpha+\sqrt{2 \lambda})=\exp (S \sqrt{2 \lambda}) \prod_{k=0}^{\infty} h\left(\lambda /(k+\alpha)^{2}\right)$, where $S$ is the negative constant defined above.

\section{The multidimensional case}

The three-dimensional (Euclidean) von Mises distribution was defined by Fisher (1953); this is the law of the colatitude of the exit point $B\left(T_{r}\right)$ for a Brownian motion with drift, starting at the origin. Hartman and Watson (1974) studied the $n$-dimensional analogue.

We use the same framework on the hyperbolic half-space $\mathbb{H}_{d}=\mathbb{R}^{d-1} \times \mathbb{R}_{*}^{+}$. Let us consider a colatitude $\varphi$ in $[0, \pi]$ such that $x_{d}=(\cosh (r)+\cos (\varphi) \sinh (r))^{-1}$. The hyperbolic Brownian motion with vertical drift $\alpha \mathbf{e}_{d}$ is constructed exactly as in Section 2: to define the law $\mathbb{P}^{(\alpha)}$ we add $\alpha x_{d} \partial / \partial x_{d}$ to the classical hyperbolic Laplacian

$$
\frac{1}{2} \Delta_{\mathbb{H}_{d}}=\frac{1}{2} x_{d}^{2} \sum_{i=1}^{d} \frac{\partial^{2}}{\partial x_{i}^{2}}+\left(1-\frac{d}{2}\right) x_{d} \frac{\partial}{\partial x_{d}} .
$$

As usual, we set $d=2 v+2$.

Proposition 10. The colatitude of the exit point of a hyperbolic Brownian motion from the sphere $S_{r}$ with radius $r$ has density

$$
\hat{\Lambda}^{\alpha}(r, \mathrm{~d} \varphi)=\frac{\sin ^{2 v}(\varphi)}{\sqrt{\pi} \Gamma\left(v+\frac{1}{2}\right)} \frac{\sinh ^{\nu}(r)}{P_{\nu-\alpha}^{(-v)}(\cosh (r))}(\cosh (r)+\sinh (r) \cos (\varphi))^{-\alpha} \mathrm{d} \varphi .
$$

This formula still has an analytical meaning for every non-integer $v>-\frac{1}{2}$.

Remark. The result agrees with Proposition 1, provided we take into account an extra factor 2 due to the different interval.

Proof. Observe first that the angular part of the hyperbolic element is the same as the Euclidean one. Therefore the angle $\varphi$ has the well-known density on $[0, \pi]$ (formula (6.2) in Kent 1977):

$$
\frac{1}{\sqrt{\pi}} \frac{\Gamma(v+1)}{\Gamma\left(v+\frac{1}{2}\right)} \sin ^{2 v}(\varphi) .
$$

Apply Girsanov's theorem to remove the drift. The space-time harmonic function is now $\exp (-\lambda t) x_{d}^{\alpha}$ with $\lambda=\alpha(\alpha-1) / 2-\alpha \nu$. Denote by $\left(\Theta_{s}\right)$ the angular part on the hyperbolic sphere $S_{1}$ with radius 1 . Under the law $\mathbb{P}^{(0)}, \Theta\left(T_{r}\right)$ is independent of $T_{r}$ and uniformly distributed on $S_{1}$. Hence the desired von Mises density is proportional to

$$
\left(\frac{1}{\cosh (r)+\cos (\varphi) \sinh (r)}\right)^{\alpha} \sin ^{2 v}(\varphi)
$$


But from in Bateman et al. (1953, 3.7 (7)), if $v>-\frac{1}{2}$,

$$
P_{\nu-\alpha}^{(-v)}(\cosh (r))=\frac{1}{\sqrt{\pi}} 2^{-v} \frac{\sinh ^{v}(r)}{\Gamma\left(\frac{1}{2}+v\right)} \int_{0}^{\pi} \sin ^{2 v}(\varphi)(\cosh (r)+\sinh (r) \cos (\varphi))^{-\alpha} \mathrm{d} \varphi .
$$

Remark. We now explain an easy way to recover the increasing 'eigenvector' such that $\frac{1}{2} \Delta_{\mathbb{H}_{d}} \varphi=\lambda \varphi$ with $\varphi(0)=1$ and $\lambda \geqslant 0$. We choose the root

$$
\alpha=v+\frac{1}{2}+\sqrt{2 \lambda+\left(v+\frac{1}{2}\right)^{2}}
$$

of the equation $2 \lambda=\alpha(\alpha-1)-2 v \alpha$. Hence the spherical mean on $S_{r}, r \rightarrow \int S_{r} x_{d}^{\alpha} \mathrm{d} \sigma$, solves the equation

$$
\frac{\partial^{2} \phi}{\partial r^{2}}+(d-1) \operatorname{coth}(r) \frac{\partial \phi}{\partial r}=2 \lambda \phi
$$

We can easily check, by means of Bateman et al. (1953, 3.7 (7)), that

$$
\int_{S_{r}} x_{d}^{\alpha} \mathrm{d} \sigma=\Gamma(1+v) 2^{v} \sinh ^{-v}(r) P_{\nu-\alpha}^{(-v)}(\cosh (r))
$$

coincides with the function $\phi_{\lambda}^{\uparrow}$ defined in Gruet (1997), the unique increasing solution of (3.3) such that $\phi_{\lambda}^{\uparrow}(0+)=1 /\left(2^{v} \Gamma(1+v)\right)$.

\section{References}

Bateman, H., Erdélyi, A. and Magnus, W. (1953) Higher Transcendantal Functions, Vol. 1. New York: McGraw-Hill.

Barndorff-Nielsen, O.E. (1978) Hyperbolic distributions and distributions on hyperbolae. Scand. J. Statist., 5, 151-157.

Casalis, M., Letac, G. and Massam, H. (1993) A decomposition for the exponential dispersion model generated by the invariant measure on the hyperboloid. J. Theoret. Probab., 6, 803-816.

Comtet, A. and Monthus, C. (1996) Diffusion in a one-dimensional random medium and hyperbolic Brownian motion. J. Phys. A, 29, 1331-1345.

Erdélyi, A. (1941) Note on Heine's integral representation of associated Legendre functions. Philos. Mag. Ser. 7, 32, 351-352.

Fisher, R.A. (1953) Dispersion on a sphere. Proc. Roy. Soc. London Ser. A, 217, 295-305.

Gertsenshtein, M.E. and Vasil'ev, V.B. (1959) Waveguides with random inhomogeneities and Brownian motion in the Lobachevsky plane. Theory Probab. Appl., 3, 391-398.

Gruet, J.-C. (1996) Semi-groupe du mouvement brownien hyperbolique. Stochastics Stochastics Rep., 56, 53-61.

Gruet, J.-C. (1997) Windings of hyperbolic Brownian motion. In M. Yor (ed.), Exponential Functionals and Principal Values Related to Brownian motion, (pp. 35-72. Madrid: Biblioteca de la Revista Matemática Iberoamericana.

Hartman, Ph. (1976) Completely monotone families of solutions of $n$th order linear differential equations and infinitely divisible distributions. Ann. Scuola. Norm. Sup. Pisa Cl. Sci. (4), 111, 
267-287.

Hartman, Ph. and Watson, G. (1974) 'Normal' distribution functions on spheres and the modified Bessel functions. Ann. Probab., 2, 593-607.

Helgason, S. (1984) Groups and Geometric Analysis. New York: Academic Press.

Ikeda, N. and Matsumoto, Y. (1999) Brownian motion on the hyperbolic plane and Selberg trace formula. J. Funct. Anal., 163, 63-110.

Jensen, J.L. (1981) On the hyperboloid distribution. Scand. J. Statist., 8, 193-206.

Kent, J.T. (1977) The infinite divisibility of the von Mises-Fisher distribution for all parameter in all dimensions. Proc. London. Math. Soc., 35, 359-384.

Lebedev, N. (1972) Special Functions and Their Applications. New York: Dover.

Mardia, K.V. (1972) Statistics of Directional Data. London: Academic Press.

Mardia, K.V. (1975) Statistics of directional data (with discussion). J. Roy. Statist. Soc. Ser. B, 37, 349-393.

Pitman, J.W. and Rogers, L.C.G. (1981) Markov functions. Ann. Probab., 9, 573-582.

Pitman, J.W. and Yor, M. (1981) Bessel processes and infinitely divisible laws. In D. Williams (ed.), Stochastic Integrals, Lecture Notes in Math. 851. Berlin: Springer-Verlag.

Rogers, L.C.G. and Williams, D. (1987) Diffusions, Markov Processes and Martingales, Vol. 2: Itô Calculus. Chichester: Wiley.

Terras, A. (1985) Harmonic Analysis on Symmetric Spaces and Applications I. Berlin: SpringerVerlag.

von Mises, R. (1918) Über die 'Ganzzahligkeit' der Atomgewichte und verwandte Fragen. Phys. Z., 19, 490-500.

Watanabe, S. (1975) On time inversion on one-dimensional diffusion processes. Z. Wahrscheinlichkeitstheorie Verw. Geb., 31, 115-124.

Watson, G.S. (1983) Statistics on Spheres. New York: Wiley.

Wendel, J.G. (1980) An independence property of Brownian motion with drift. Ann. Probab., 8, 600601.

Yor, M. (1980) Loi de l'indice du lacet brownien et distribution de Hartman-Watson. $Z$. Wahrscheinlichkeitstheorie Verw. Geb., 53, 71-95.

Yor, M. (1992) Some aspects of Brownian Motion. Part I: Some Special Functionals. Basel: Birkhäuser.

Yor, M. (1993) Further results on exponential functionals of Brownian motion. Preprint.

Received February 1999 and revised November 1999 